Logic and Logical Philosophy

Volume 14 (2005), 145-163

DOI: $10.12775 /$ LLP.2005.011

\title{
Anna Sierszulska
}

\section{MEINONGIAN EXTENSIONS OF PREDICATES}

\begin{abstract}
The paper analyses the contemporary notion of an extension of a predicate from the perspective of semantics typical for Meinongian logics, and in opposition to the traditional notion of extension. This leads to a discussion of the types of properties that can be predicated about objects as belonging to the sets of properties ascribed to them, and such that can be predicated about them only 'externally'. It is also problematic in which sense nonexistent objects possess the properties ascribed to them. The concluding remarks concern some issues related to the truth of predications about nonexistent objects, which is the basic condition to admit them as belonging to the extensions of predicates in the first place.
\end{abstract}

Keywords: Meinong, nonexistent objects, extensions in predicate logic

A Meinongian semantics involves either two notions of extension, or just one very broad notion. The 'broad' notion of extension corresponds to Meinong's uniform treatment of all objects as bundles of properties, regardless of their being or non-being. The Meinongian domain contains not only abstract and merely nonexistent, but also impossible objects, and thus the extensions of predicates become quite something else than ordinary extensions.

It should be observed that in modal Meinongian logics possible worlds are not constituted solely of would-be existent objects. The objects belonging to the extensions of predicates in each world show some diversity as to their existential status. An extension is, therefore, no longer a set of ordinary existent individuals (in each possible world), because nonexistent objects may be its members as well. Lambert explains that this is characteristic of the contemporary notion of extension, which differs from the traditional notion. 
Contemporary talk about extensionality has somewhat distorted the traditional notion. For example, the extension of a general term, in the contemporary parlance originating in Carnap's classic Meaning and Necessity, say, 'winged horse', is customarily taken to be simply the set of objects it is true of, as opposed to the intension of that term (a property, a function, etc.) So construed it would comprise, for instance, merely possible objects (such as Pegasus) as well as actual objects (such as Meinong). It is not surprising therefore, that traditional and current talk of extensions can yield different verdicts about whether a given theory of predication violates the principle of extensionality, a principle which demands that coextensional general terms be everywhere substitutable salva veritate. (...) in the contemporary mode, Meinong's logic may be every bit as extensional as 'extensional logic'; in the traditional mode, it certainly is not... ${ }^{1}$

Therefore, if the round square is an object in our Meinongian domain and we accept that the sentence 'The round square is round' is true, then the round square belongs to the extension of the predicate in question. We have an extensional semantics always when properties are defined extensionally as sets of objects, no matter if these objects are existent or possible or impossible. The distinctive feature of an extensional semantics is that coextensional properties cannot be distinguished, as opposed to intensional semantics, making a difference between the senses of expressions. It is a specific characteristic of a Meinongian semantics that it can be intensional even though it does not introduce possible worlds. We will see on the example of Jacquette's semantics that if the broad extensions of predicates are taken into account so that they include all intentional objects referred to in attitude and nonexistence contexts, this results in an intensionally sensitive semantics. Zalta's semantics, on the other hand, is essentially intensional, since all properties are intensionally distinct by acquaintance, prior to defining their extensions.

Parsons has only one broad notion of extension which includes nonexistent objects, such as he considers to be legitimate ones, i.e. present in the linguistic practice. He admits that the primary extension of a predicate consists of real objects, but these are not all the objects belonging to the full extension of the property for nuclear properties. We can suppose that the

\footnotetext{
${ }^{1}$ Karel Lambert, Meinong and the Principle of Independence, Cambridge University Press, 1983, pp. 64-65.
} 
extension of a property may sometimes consist only of real objects (e.g. the property of being existent), or entirely of nonexistent objects (the property of being incomplete).

(...) whereas the primary extension of a nuclear property is a subclass of the real objects, such a property will also be possessed by certain unreal objects. The class of all objects which possess a property will be called the full extension of that property. ${ }^{2}$

The identity conditions for properties on Parsons' intensional logic are defined according to their full extensions and not just their ordinary extensions. The extensions of identical properties must be indentical even with respect to the membership of impossible objects.

The notion of nuclear property being used here is something like C. I. Lewis's notion of the comprehension of a predicate. Lewis identified this with the set of all possible objects to which the predicate is applicable. Our identity conditions for nuclear properties are actually even tighter than this, for nuclear properties are identical only if they coincide for impossible objects as well. (This may make them closer to Lewis's notion of the intension of a term $(\ldots)) .^{3}$

Naturally, the same property may have different extensions relative to worldtime pairs, but since Parsons identifies world-time pairs with different interpretations of the language that agree with the basic interpretation "about which predicates stand for which properties" 4 , we can speak about the identity of nuclear properties as the full extensions of these properties on a single interpretation.

In opposition to those Meinongian logicians who admit nonexistent objects into their basic domains of interpretation, Paśniczek has just the traditional notion of extension, because his basic domain contains only ordinary existing individuals and properties are defined on this domain exclusively. The nonexistent M-objects are constructed out of sets of individuals which constitute the ordinary extensions of properties. There are, strictly speaking, no nonexistent objects on full rights in Paśniczek's semantics. The M-objects that can be identified with existents turn out to be ordinary individuals, but there are no individuals in the domain to be identified with nonexistent M-objects. So Paśniczek's notion of extension is untypically traditional.

\footnotetext{
${ }^{2}$ T. Parsons, Nonexistent Objects, Yale University Press, 1980, p. 79.

${ }^{3}$ T. Parsons, Nonexistent Objects, p. 75.

${ }^{4}$ T. Parsons, Nonexistent Objects, p. 99.
} 
In contrast to Paśniczek's austere proposal, where the basic domain includes only existent individuals, Jacquette's domain contains every conceivable entity. This is a true Meinongian domain, one might say.

The model consists of three ordered components: a domain $\mathrm{D}$, an interpretation I on the domain, and a truth valuation of propositions under the interpretation $\mathrm{V},\langle\mathrm{D}, \mathrm{I}, \mathrm{V}\rangle .(\ldots)$ Domain $\mathrm{D}$ thus contains an object corresponding to every grammatically welldefined term, constant, predicate, functor, definite descriptor, and lambda abstract. Some of the objects are sets of objects, where every condition on any objects determines a set. (...) A nonstandard version of Zermelo-Fraenkel set theory drives the semantics. ${ }^{5}$

The membership in the extension sets of properties is non-standard in Jacquette's semantics, because there are many nonexistent objects which he considers to be undetermined as to their property possession. Therefore, properties are identical on his view only if the same objects belong to their extensions and the same objects are also undetermined with respect to these properties. Since the property sets contain both existent and nonexistent entities, he calls them intensions instead of extensions of properties. This use of the notion of property intension does not coincide with the standard use of this notion, which is reserved for possible worlds options of the extensions of properties, where the property intensions can actually differ in the membership of objects from one possible world to another. No such thing happens when we have a one-world semantics. We rather have a broad extension of a predicate in this case, i.e. an extension of a predicate that contains both ordinary and nonexistent objects. But it is true that Jacquette's semantics is intensional, i.e. sense preserving, because the strict identity conditions for properties, concerning also the set membership of intentional and nonexistent objects, prevent properties from becoming identical just on the basis of the extensions that consist of existent objects.

The predicate semantics for the object language of the logic can now be set-theoretically defined.(...) This provides an intensional counterpart of the identity of a property with the existent and nonexistent objects in the intensions of corresponding predicates, parallel to the identity of a property with the existent objects in the extensions of corresponding predicates in standard extensional semantics. The intension of a predicate is non-Fregean,

\footnotetext{
${ }^{5}$ D. Jacquette, Meinongian Logic: The Semantics of Existence and Nonexistence, Walter de Gruyter \& Co, Berlin-New York, 1996, p. 103.
} 
though indirectly related to Frege's property-'senses' by virtue of the identity conditions for objects, determined by their Soseine, or associated unordered sets of nuclear constitutive properties. ${ }^{6}$

Jacquette's intension of a predicate, or what I call its broad extension, captures the 'sense' of a property by means of including intentional objects, which play the role of linguistic meanings in attitude contexts. Thus a property is defined by all the meaning contexts in which it can be truly predicated. We may assume that if two properties are impossible to distinguish in all meaning contexts, then they are intensionally identical. The diagrams for property membership presented by Jacquette (p.109) show that for contradictory properties $\mathrm{P}$ and -P their broad extensions will have an overlapping common part that corresponds to the joint membership of impossible objects in their extensions, and there are also some objects that do not belong to either of the two sets, because they are undetermined as to the possession of this property or its converse.

A Meinongian semantics may have to introduce nonexistent objects into the extensions of predicates, or, like Zalta's, it may introduce double extensions. Zalta's A-objects do not exemplify properties, they only encode them and for this reason, while existing objects belong to ordinary extensions of predicates, the 'abstract' objects belong to their encoding extensions.

Since encoding is a kind of predication, we may regard properties as having a second kind of extension. In addition to having an exemplification extension, which consists of just those individuals that exemplify the property, they also have an encoding extension, which consists of just those individuals that encode the property. ${ }^{7}$

We can see the parallel within Zalta's theory between two kinds of extension and two kinds of denotation of expressions. This is related to the fact that the domain contains individuals of two kinds: existent and 'abstract' individuals. Zalta makes it clear that his denotations do not correspond to ordinary exemplification extensions, because in that case his system would be purely extensional, its intensionality would be lost.

In what follows, we shall not use the method of intension and extensionUthe terms of our language receive only denotations,

\footnotetext{
${ }^{6}$ D. Jacquette, Meinongian Logic: The Semantics of Existence and Nonexistence, p. 108.

${ }^{7}$ E. Zalta, Intensional Logic and the Metaphysics of Intentionality, Massachussets Institute of Technology Press, 1988, p. 29.
} 
relative to an interpretation of the language and an assignment to the variables. Moreover, Substitutivity is preserved intact in our logicÙ:there are no denoting terms, or contexts, for which it fails. Truth is always preserved when terms having the same denotation are substituted for one another. This last fact could be a source of confusion, if one were inclined to think of the denotation of a term as corresponding in some way with the extension of a term. For then one could describe our system as purely extensional $(\ldots)^{8}$

The denoting expressions of the language denote either existent objects or 'abstract' sense-objects, depending on the context. The former belong to the exemplification extensions of predicates and the latter to the encoding extensions of predicates within each 'possible world'. Zalta's denotations of both kinds are not relative to world-time pairs, because only the extensions of predicates change from one world to another. As we have already seen above, names and definite descriptions are rigid designators. Their denotations are always A-objects in the case of nonexistents. Otherwise, Aobjects are used for explanatory purposes in propositional attitude contexts, on which occasions terms denote such sense-objects instead of ordinary individuals. The immediate consequence of this situation for the truth value of propositions is that the membership of existent individuals in the exemplification extensions of predicates need not coincide with the membership of the A-objects - denoted instead of these individuals in attitude contexts - in the encoding extensions of the same predicates. An existing individual will typically exemplify more properties than the properties encoded by a corresponding A-object in a certain attitude context. Thus 'abstract' objects are not admitted into the ordinary extensions of predicates together with existent objects, but there is a second notion of extension concerning 'abstract' objects specifically, the encoding extension, which is important for the functioning of Zalta's semantic system.

One might ask why it is important to make any distinctions among the properties of existent and nonexistent objects. The reason for this is that Meinong's principle of free assumption of an object's Sosein (the set of properties that constitute an object) results in some paradoxes without such distinctions. Some examples are: 'the existent golden mountain', 'the thought which is a thought about itself', 'the object which is a Meinongian object'. There are two distinctions designed to prevent the paradoxes: of

\footnotetext{
${ }^{8}$ E. Zalta, Intensional Logic and the Metaphysics of Intentionality, p. 9.
} 
two kinds of properties and of two kinds of predication, both originally introduced by Meinong's student, Ernst Mally. The distinction between two kinds of properties, described by Mally as 'formale' and 'ausserformale', was in fact accepted by Meinong himself as he defended his assumptions principle against Russell's attacks. Meinong calls them 'konstitutorische' and 'ausserkonstitutorische' - to give the distinction more explanatory value, and the distinction is translated by Findlay as a distinction between 'nuclear properties' - those belonging to the Sosein of an object, and 'extranuclear properties' - which are external to the Sosein of an object, such as being existent, impossible, incomplete. This distinction has been incorporated into the semantic theories of Terence Parsons, Richard Routley and Dale Jacquette.

The second distinction, between predications of two kinds, originates from Mally's terms 'determinieren' and 'erfüllen', which mean, according to Findlay, being determined by a set of properties and satisfying the properties. The distinction between two kinds of predication has been utilized in a few Meinongian semantics under different names. There is the 'internal' and 'external' predication of Hector-Neri Castaneda and Jacek Paśniczek, the 'constituting' and 'exemplifying' of William Rapaport, and the 'encoding' and 'exemplifying' of Edward Zalta. The aim of such a distinction is to mark the difference between the ordinary exemplification of properties by real objects, and the possession of properties by semantic objects, since the latter cannot really exemplify any properties that are ascribed to them.

There has been some discussion around these two distinctions concerning such questions as whether one of them is more appropriate than the other, and whether they are intertranslatable so that one can be explained in terms of the other. The distinctions seem to reflect two different approaches to predication, which brought to their extreme could be expressed by (a) the strangeness claim about objects, and (b) the strangeness claim about properties.

(a) the strangeness claim about objects: NO PROPERTIES CAN BE PROPERLY PREDICATED OF SOME OBJECTS (WHICH ARE NONEXISTENT OBJECTS) $\Rightarrow$ SOME OBJECTS ARE NOT ORDINARY OBJECTS OF PREDICATION

(b) the strangeness claim about properties: SOME PROPERTIES (EXTRANUCLEAR) CANNOT BE PROPERLY PREDICATED OF ANY OBJECTS $\Rightarrow$ SOME PROPERTIES ARE NOT ORDINARY PROPERTIES.

According to (a), some objects can only encode or include properties, but they cannot exemplify properties, so they are not objects on equal rights 
with ordinary objects. According to (b), some properties do not constitute the nature of objects, so they are not ordinary (nuclear) properties.

The situation is further complicated by the fact that the strangeness claims, both about objects and about properties, are not applied consistently. It is said that semantic objects do exemplify some properties, like being abstract objects, or being incomplete objects. Such 'extranuclear' properties, in turn, although normally non-constitutive, can have their nuclear 'watered-down' versions. So most Meinongian semantics make use more or less explicitly of both distinctions and they try to describe the problem in terms of both, if only by polemics.

In Parsons' logic of nonexistent objects, apart from the nuclear-extranuclear distinction, which plays an important role in the theory, the distinction between 'having' and 'including' properties is also mentioned.

The obvious thing to do here is to make a distinction between a property's being in the set correlated with an object, and the object's having the property. In the former case, let us say that the object includes the property. Presumably, real objects have exactly the properties they include, but for others there will be a divergence. (...) When applied to language, this theory has the consequence that predication is ambiguous: $F a$ can mean either that $a$ has Fness or that $a$ includes Fness. ${ }^{9}$

While real objects have all the properties they include, fictional objects need not have any of the properties they include, or maybe they can have some and only include others. Parsons is quite precise about his preferences, but his 'orthodox' theory says that fictional objects have extranuclear properties and only include nuclear ones. In the end, he decides that at least the treatment of all nuclear properties should be uniform for all objects.

But for nuclear properties no such divergence seems appropriate. Perhaps fictional objects have all the nuclear properties that they include, as on the present theory, or perhaps they have none $(\ldots)^{10}$

Thus, both real and fictional objects of Parsons 'have' all their nuclear properties, and the distinction between two kinds of predication is dropped on this level. But is it dropped on all levels? It may be quite revealing to quote a passage from Cocchiarella.

\footnotetext{
${ }^{9}$ T. Parsons, Nonexistent Objects, p. 171.

${ }^{10}$ T. Parsons, Nonexistent Objects, p. 172.
} 
The possession of an extranuclear property is in that case correlated with the orthodox Russellian predication of a higher order property or relation. Such a difference in logical type and level in the representation of nuclear and extranuclear properties and relations, needless to say, might well be thought to be indicative of an underlying structural difference in Parsons's theory between two types, or forms of predication, viz., a Meinongian predication of nuclear properties, and a Russellian predication of extranuclear properties and relations. ${ }^{11}$

Parsons' is a second order logic, and, at least according to his 'orthodox' theory, his extranuclear predicates signify second order properties, e.g. 'being existent' is a property predicated of a set of properties that represents an individual. Extranuclear predication is higher order and always ordinary - the extranuclear properties are genuinely possessed by the set of properties in question. The nuclear predication, in agreement with what Parsons says, is sort of indifferent to the issue of the object's actually having the properties or not. The primary relation, also for existent objects, is that of the properties being included in the set representing an individual. Whether the object really has them, or does not have them, seems to be another matter. So we can see that, in a way, two kinds of predication are involved in Parsons' theory.

Zalta is in favour of two kinds of predication, and against the nuclearextranuclear distinction. He emphasises very much the fact that 'abstract' objects do not exemplify properties in the same way real objects do.

Things that exemplify the property of being a detective exist, have a location in space and time, are made of flesh and bones, think, solve crimes, and so on, whereas things that just encode the property of being a detective are abstract and do not exemplify any of these characteristics. They might exemplify these properties according to their respective stories, but this is not the same as exemplifying them simpliciter. ${ }^{12}$

Zalta's abstract A-objects encode most of their properties. However, Aobjects can also exemplify some properties, and incidentally, these are exactly the properties which are called extranuclear according to the distinction he rejects. For this reason, his theory can be supposed to contain the

\footnotetext{
${ }^{11}$ Nino Cocchiarella, "Meinong Reconstructed versus Early Russell Reconstructed". In: Logical Studies in Early Analytic Philosophy, Ohio State University Press, 1987.

${ }^{12}$ E. Zalta, Intensional Logic and the Metaphysics of Intentionality, p. 17.
} 
nuclear-extranuclear distinction implicitly. He could avoid this outcome, if he tried to be more consistent in applying the non-exemplification principle to 'abstract' objects, so that they would not be able to exemplify any properties at all. As it is, it seems (which is pointed out by Jacquette) that there is no clear principle when and which properties can be exemplified by 'abstract' objects.

The limitation of Zalta's informal principle is that while it rules out exemplification by an abstract object when no existent object exemplifies the properties an abstract object encodes, it does not work in the opposite direction to determine when an abstract object exemplifies the properties it encodes. ${ }^{13}$

There is only the negative principle when the properties encoded cannot be exemplified, namely, when the A-object is not an auxiliary meaning-object that can be identified with an existent individual. The properties encoded by a nonexistent object cannot be exemplified. But which properties are merely encoded by a nonexistent object and not exemplified by this object? It seems that these are the extranuclear properties. Jacquette also makes the objection, that strictly speaking, if Zalta wanted to be consistent in his theory as independent from the other distinction, 'abstract' objects should not exemplify any properties, even the property of being abstract.

It is not enough for Zalta to reform his use of what might now be called extranuclear predicates 'E!' and 'A!'. Even if he were to agree that abstract objects can only encode properties, and no abstract object exemplifies or really has a property, and write ' $x \mathrm{~A}$ !' or ' $\sim x \mathrm{E}$ !' instead of 'A! $x$ ' or ' $\sim \mathrm{E} ! x$ ', the philosophical problem remains. (...) To make a special case for properties like 'abstract' and 'nonexistent' is tacitly to rely on a distinction between constitutive and nonconstitutive or nuclear and extranuclear properties, which categories of properties Zalta indeed refers to respectively as 'nontheoretical' and 'theoretical'. ${ }^{14}$

Zalta could change his notation and represent 'being abstract' as ' $x$ A!' $x$ encodes $\mathrm{A}$, instead of 'A! $x$ ' $-x$ exemplifies $\mathrm{A}$, but we have to agree with Jacquette that the philosophical problem remains. Does an abstract object exemplify the property of being abstract or does it encode this property? I would be inclined to think that abstract objects exemplify no properties at all. Yet they really are abstract objects, aren't they? Making use of Zalta's expression, 'being abstract' is a 'theoretical property' and so it may be the

\footnotetext{
${ }^{13}$ E. Zalta, Intensional Logic and the Metaphysics of Intentionality, p. 37.

${ }^{14}$ D. Jacquette, Meinongian Logic, p. 26-27.
} 
most proper to understand it as a higher order property of sets of properties representing objects. Perhaps we could simply say that it is not predicated at the level of ordinary properties on which the 'encoding -exemplifying' distinction operates.

Some logicians, like Kit Fine ${ }^{15}$, Jaquette, or Paśniczek, think that the two distinctions are either intertranslatable, or that it is possible to get rid of one of them in favour of the other. Jacquette thinks that the nuclearextranuclear distinction is more basic and the dual predication can be reduced to this distinction, although his attempt to do so is not quite successful due to some misconceptions concerning Zalta's theory, which he is trying to reduce.

The reduction of the dual copula or dual modes of predication to to the nuclear-extranuclear property distinction is easy to accomplish, since the two predication modes arise entirely in connection with whether or not an object has the extranuclear property of existence. ${ }^{16}$

There are also voices in defense of the distinction between two kinds of predication, which appears to be more clear and more elegant. Paśniczek, for example, proposes internal and external predication, with constitutive properties predicated internally. ${ }^{17}$ The innovation is that it differs from object to object which properties are those predicated internally or externally, so there is no preset list of properties of each kind, as in the case of nuclear and extranuclear properties. This solution combines the two distinctions, but it differs from Zalta's encoding-exemplifying division. There is no encoding for existent objects, and no exemplification for ordinary properties of abstract objects on Zalta's theory, while Paśniczek's internal and external predications apply to all objects indiscriminately.

In opposition to the proponents of intertranslatability, Zalta thinks that the translation between the two distinctions is impossible, at least on the ground of his theory, because the same property can be both exemplified and encoded depending on whether the object is ordinary or 'abstract.' ${ }^{18}$ There is indeed a certain lack of correspondence involved here, as the two distinctions originate from the two different approaches to predication, which

\footnotetext{
${ }^{15}$ Kit Fine, "Critical Review of Parsons' Nonexistent Objects", Philosophical Studies 45.

${ }^{16}$ D. Jacquette, Meinongian Logic, p. 17-18.

${ }^{17}$ J. Paśniczek, The Logic of Intentional Objects. A Meinongian Version of Classical Logic, Kluwer, Dordrecht, 1998, p. 122.

${ }^{18}$ E. Zalta, "On Mally's Alleged Heresy: A Reply", online.
} 
I describe above as the strangeness claim about objects and the strangeness claim about properties.

The mutual interrelations between 'nuclear-extranuclear' and 'encodedexemplified' distinctions can be understood as follows:

If $\mathrm{P}$ is AN EXTRANUCLEAR PROPERTY THEN P IS EXEMPlified.

IF $\mathrm{P}$ IS EXEMPLIFIED THEN $\mathrm{P}$ IS AN EXTRANUCLEAR PROPERTY, OR A NUCLEAR PROPERTY OF A REAL OBJECT.

IF Q IS ENCODED THEN Q IS A NUCLEAR PROPERTY.

IF $Q$ IS A NUCLEAR PROPERTY THEN $Q$ IS ENCODED BY AN ABSTRACT OBJECT OR EXEMPLIFIED BY A REAL OBJECT.

Therefore, We COnClude:

ENCODED $=>$ NUCLEAR, BUT NOT CONVERSELY; -NUCLEAR: ENCODED OR EXEMPLIFIED

EXTRANUCLEAR $=>$ EXEMPLIFIED, BUT NOT CONVERSELY;

EXEMPLIFIED: EXTRANUCLEAR OR NUCLEAR

Judging from the above one may have some doubts whether these two distinctions are really counterparts of each other, or even if they are indeed intertranslatable in any straightforward way. Perhaps it is useful to make both distinctions, for they concern slightly different issues.

Another option might be to assume that all properties possessed by objects, both by way of encoding and exemplification, are ordinary properties, coinciding with what is called 'nuclear' properties. The 'extranuclear' properties would not have to be considered as properties of objects at all. They could be substituted by logical operators of some kind whenever there is a need to express the idea that the objects we speak about appear in a certain context as existent, fictional, incomplete, etc.

Alternatively, one could assume that such properties are higher order properties, or belong to a metalanguage, since they are usually the properties not of objects but of sets of properties that constitute objects. Thus, being contradictory, nonexistent and incomplete are not properties of the object the round square, but of the set of properties being round, being a square. Someone might object that being a fictional, abstract, or mythological object, on the other hand, does not apply to sets of properties, but to the objects, like Pegasus. Yet we can still argue that such properties belong to 
the meta-discourse. In consequence, it might be possible not to employ the slightly awkward distinction between nuclear and extranuclear properties.

As to the other distinction, between two kinds of predication, there is no absolute need to employ it either, because the paradoxes may be taken care of by means of introducing logical operators for special contexts or higher order predicates for 'external' properties. All the same, I think that distinguishing between exemplification of properties by real objects and mere encoding, or including, of properties by abstract objects, has some explanatory value and may contribute to avoiding many misunderstandings on the part of more Russellian-minded philosophers.

As it has been mentioned above, the contemporary notion of extension puts no existential requirements upon the objects belonging to the extensions of predicates, but just a liberal requirement that predications of the properties in question should be true about these objects which belong to the extensions of predicates. Admitting nonexistent objects into a Meinongian domain and claiming that predications about them may be true, requires a formal justification, which is provided by so called 'principle of independence'. Like most of the logic related to Meinong's philosophy, the principle has its origin in the work of Ernst Mally. In a book devoted to this principle, Karel Lambert presents its two formulations, a strict and a looser version. The strict version makes use of the notions of Sosein and of nuclear (constitutive) properties, in order to express the idea that an object may have a Sosein consisting of a certain set of nuclear properties, even though the object does not possess being.

The strict version says that the principle:

"There are nuclear properties $\mathrm{P} 1, \mathrm{P} 2 \ldots$ such that the set of $\mathrm{P} 1$, $\mathrm{P} 2$... attaches to s;

So, s has being"

- is invalid. ${ }^{19}$

The loose version, in turn, says that the principle: "There is a property $\mathrm{P}$ such that $\mathrm{P}$ is possessed by $\mathrm{s}$;

So, s has being"

- is invalid.

\footnotetext{
${ }^{19}$ Karel Lambert, Meinong and the Principle of Independence, p. 28.
} 
Lambert explains the difference between these two versions as follows: This looser version of the principle of independence differs from the strict version in two key respects. First, no mention of a set of properties (a Sosein) attaching to an object occurs in instances of the looser version. Second, the properties satisfying particular instances of the looser version can be extranuclear in contrast to the strict version. $(\ldots)^{20}$

Since the looser version is simpler and also affords continuity between the authorities and the current work, it will henceforth be adopted. This means leaving behind the nuclear-extranuclear property distinction vital to Meinong's theory. ${ }^{21}$

It seems that the advantage of the looser version is that predication may be true about an object even when the only property ascribed to it is a single, extranuclear property. So the object need not be constituted properly to be regarded as an object by Lambert, because his main concern is preserving the truth of predications for otherwise irreferential expressions, which is characteristic for his variety of free logic.

Lambert classifies free logics as positive, which are those according to which the predications concerning nonexistent objects are true, negative free logics - with only false predications of nonexistents, and neuter, on which such predications are truthvalueless. ${ }^{22}$ His own view is that some predications about nonexistent objects can be true, although such terms are irreferential. In the Russellian tradition, the 'is' of predication implies the 'is' of being, but Russell differentiates the 'is' of predication and the 'is' of identity. Identity statements about nonexistents may be true, even though predications must be false. The Meinongian tradition, represented also by Lambert, treats the 'is' of identity and of predication on equal rights, since both "The round square is the round square" and "The round square is round" are true sentences. None of these true sentences implies the 'is' of being, though.

However, terms such as 'the round square' do not stand for beingless objects, according to Lambert, since in free logic there is no need for beingless objects, it is sufficient to admit irreferential terms. ${ }^{23}$ He remarks that, even though there are some free logicians who hold that such terms refer to

\footnotetext{
${ }^{20}$ Karel Lambert, Meinong and the Principle of Independence, p. 29.

${ }^{21}$ Karel Lambert, Meinong and the Principle of Independence, p. 30.

${ }^{22}$ Karel Lambert, Meinong and the Principle of Independence, p. 116.

${ }^{23}$ Karel Lambert, Meinong and the Principle of Independence, p. 76.
} 
nonexistent objects, none of them quantifies over such objects. ${ }^{24}$ One might wonder why it should be such a bad idea to quantify over the objects which legitimately belong to the extensions of predicates. That is because, on the standard view, this marks ontological commitment to such entities.

As we know, the most typical feature of Meinongian logics is supposed to be quantifying over nonexistent objects. But those of the Meinongian-style logicians who actually quantify over nonexistent objects, hardly ever agree that, by this fact, they are committed to the existence of what in principle does not exist. They say that 'there are' nonexistent objects, although none of them have either existence or any kind of being. The mystery of this paradoxical position lies in the distinction between two senses of 'there is'. One of these senses is expressed by the ordinary existential quantifier ranging over all objects in the Meinongian domain. The typical reading that it receives has nothing to do with an object's existential status:

$\exists x-x$ BELONGS TO THE DOMAIN, OR THERE IS AN OBJECT $x$ IN THE DOMAIN

The other sense of 'there is', the one which is standardly supposed to be expressed by existential quantifier, and which affirms an object's actual existence or, more generally, its being, is conveyed in Meinongian logics by a special existence predicate:

E! $x-x$ HAS BEING, OR AN OBJECT $x$ IN THE DOMAIN HAS BEING

The question appears what is 'E!'. It is not a second existential quantifier, though it seems to play a similar role. It is called a predicate, which sounds fine when it is a second order predicate. But it is also employed in first order logics, where it must certainly be regarded as a very special predicate, because existence is definitely not an ordinary property of objects.

In any case, it is by means of 'E!' that the ontological commitment usually associated with the existential quantifier is expressed. For instance, Jacquette writes:

The 'existential' quantifier with bound variables ranging over existent and nonexistent objects in the Meinongian domain cannot serve as a criterion of ontological commitment. Instead the test must be to examine canonical formulations of a theory for occurences of extranuclear existence predications to determine which objects the theory claims to exist. ${ }^{25}$

\footnotetext{
${ }^{24}$ Karel Lambert, Meinong and the Principle of Independence, p. 97.

${ }^{25}$ D. Jacquette, Meinongian Logic, p. 95.
} 
Thus, Quine's criterion as to which objects a theory is committed, is abandoned. No singular terms can be eliminated by means of Russellian descriptions and so it is not enough to be the value of a bound variable to have being. Being must be explicitly stated by means of the existential predicate in order to determine to which objects it pertains. This is a requirement not only with respect to real existent objects, but with respect to any object that is claimed to have being of any kind.

$(\ldots)$ the extranuclear existence property 'E!' is intended to subsume both of Meinong's existence and subsistence categories. ${ }^{26}$

Once such a proclamation of being is made, Jacquette is glad to admit that the ontological commitment of a theory has been stated explicitly and beyond any doubts. Which is good, because it enables us to see clearly which theories are committed to which entities.

A theory about unicorns on this analysis turns out to be ontologically committed to the existence of unicorns, even though no unicorns exist. ${ }^{27}$

It does not matter entirely whether entities of a certain kind really exist in the world or not. If the theory claims their existence, it is committed to them, as the theory of 'ether' was committed to ether, for example, even though it was false. Jacquette thinks that this approach is useful in application to certain scientific theories that are committed to nonexistent theoretical entities like the ideal gas or the frictionless surface or infinitesimals.

Also in Zalta's theory, apart from the usual existential quantifier, there is the existence predicate.

Clearly, there are two notions of existence that may be distinguished in our logic. One is expressed by the predicate 'E!'. The other is expressed by the quantifier ' $\exists$ ', even though we have used the words 'some' and 'there is' to read this symbol. ${ }^{28}$

Consequently, because the quantifiers do not carry any commitment obligation, Zalta may quantify over the whole domain including 'abstract' objects.

The nonexistence of an object doesn't diminish its status as an object over which we may quantify. ${ }^{29}$

\footnotetext{
${ }^{26}$ D. Jacquette, Meinongian Logic, p. 116.

${ }^{27}$ D. Jacquette, Meinongian Logic, p. 69.

${ }^{28}$ E. Zalta, Intensional Logic..., p. 102.

${ }^{29}$ E. Zalta, Intensional Logic. ., p. 68.
} 
A generous policy concerning quantification is assumed by Parsons as well, whose existence predicate is used by him to formulate explicitly an axiom according to which there are such objects that no such objects exist, paraphrasing Meinong's well known saying "there are objects that there are no such objects".

$(\ldots)$ it is explicitly assumed that variables may range over all objects, not just those that exist. There is an extranuclear predicate of existence, written 'E!', and there are axioms which entail that there are objects that don't exist: $(\exists x) \sim \mathrm{E} ! x^{30}$

This is in agreement with what we are told at the beginning of his book, that the theory presented will claim that there are, in fact, nonexistent objects:

The theory given below will say that there are unicorns, there is such a thing as Pegasus, etc., but that none of these exist. ${ }^{31}$

The only problem is with the meaning of the phrase 'there are' in the existence-neutral sense, which I characterize above as 'belonging to the domain'. Doesn't it really imply any kind of being at all? Parsons says that he does not know and has no intention of taking sides on this issue.

Russell objected that if there are objects that do not exist, they have to have some other kind of being. I have never been able to find more than a terminological issue here. If there is an issue about whether nonexistent objects have some kind of being, I intend to remain neutral on the issue. ${ }^{32}$

Some people think that there is an issue, though, and that either there are no nonexistent objects or else they must have some other kind of being. For this reason, they find it safer not to quantify over such objects, or to quantify over them very carefully, so as not to suggest more than they in fact want to assert. The substitutional reading of quantification is an example of such a strategy.

(...) some proponents of substitutional quantification assume that there are names that fail to refer but which nonetheless appear in true sentences of the form ' $\mathrm{N}$ is an A', and then they hold that some sentences of the form 'There is an A' are true even though no object is an A. ${ }^{33}$

\footnotetext{
${ }^{30}$ T. Parsons, Nonexistent Objects, p. 155.

${ }^{31}$ T. Parsons, Nonexistent Objects, p. 11.

32 T. Parsons, Nonexistent Objects, p. 10.

${ }^{33}$ T. Parsons, Nonexistent Objects, p.12.
} 
In other words, if one accepts certain instances of predication as true, one also has to agree that certain instances of quantification are acceptable, without accepting that there are, in any sense, the objects quantified over. On a substitutional reading of the existential quantifier, we accept that at least one instance of the substitution of a name from a certain inventory of names must be true. But we do not assume anything about the existence of the objects signified by the names we substitute. In relation to substitutional quantification two issues are important - as pointed out by Susan Haack ${ }^{34}$. First, if it is assumed, and it is assumed on a Meinongian theory, that singular terms are not eliminable by means of Russell's theory of descriptions, then quantification over variables need not be the only way to express ontological commitment to objects, because singular terms can perform this task by either denoting existent entities or not. It follows from this observation that we can agree to have non-commital quantification over all entities in a Meinongian domain and there is no real need for substitutional quantification. Second, substitutional quantification only postpones ontological questions, shifting them from the quantifiers to the names. Now we can make a choice which names we admit to be substituted for the variables. A Meinongian semantic theory will obviously admit names of nonexistent entities. But we may be surprised to notice that the truth of instances of subsitution of such names for the variables quantified over depends in the first place upon whether we accept that predications concerning the objects signified by these names can be true. In a Meinongian semantics, it is accepted that every predication is true if a given property belongs to the set of properties ascribed to the object in question, regardless of its existence or nonexistence. And since the truth of predications determines the extensions of predicates, not only existent objects belong to Meinongian extensions of predicates.

\section{References}

[1] Cocchiarella, N., "Meinong Reconstructed versus Early Russell Reconstructed". In: Logical Studies in Early Analytic Philosophy, Ohio State University Press, 1987, pp. 119-151.

[2] Fine, K.,"Critical Review of Parsons' Nonexistent Objects", Philosophical Studies 45: 95-142, 1984.

[3] Haack, S., Philosophy of Logics, Cambridge University Press, 1978,

${ }^{34}$ Susan Haack, Philosophy of Logics, Cambridge University Press, 1978, pp. 46-50. 
[4] Jacquette, D., Meinongian Logic: The Semantics of Existence and Nonexistence, Walter de Gruyter \& Co, Berlin-New York, 1996.

[5] Lambert, K., Meinong and the Principle of Independence, Cambridge University Press, 1983.

[6] Parsons, T., Nonexistent Objects, Yale University Press, 1980.

[7] Paśniczek, J., The Logic of Intentional Objects. A Meinongian Version of Classical Logic, Kluwer, Dordrecht, 1998.

[8] Zalta, E., Intensional Logic and the Metaphysics of Intentionality, Massachussets Institute of Technology Press, 1988.

[9] Zalta, E., "On Mally's Alleged Heresy: A Reply". Online: http://mally.sta nford.edu/jacquette.pdf

\author{
Anna Sierszulska \\ Institute of Philosophy \\ Jagiellonian University \\ ul. Grodzka 52 \\ 31-044 Kraków \\ Poland \\ a.sierszulska@iphils.uj.edu.pl
}

